\title{
«Der grösste Gesundheitsgewinn kann durch Gesundheitsförderung und Prävention erzielt werden»
}

Er prägte die Schweizer Gesundheitspolitik während knapp zwei Jahrzehnten an vorderster Front mit: Professor Thomas Zeltner, Arzt und Jurist, stand dem Bundesamt für Gesundheit BAG von 1991 bis Ende 2009 als Direktor vor. Nach einem fulminanten Schlussspurt mit «Schweinegrippe» und Dutzenden von Abschlussinterviews gewährten wir dem nach wie vor Vielbeschäftigten eine Verschnaufpause, bevor wir mit ihm auf seine Amtszeit zurückblickten.

Interview: Anna Sax und Bruno Kesseli
Herr Zeltner, Sie kommen eben von einem Aufenthalt an der US-Eliteuniversität Harvard zurück. Lancieren Sie bereits Ihre nächste Karriere?

Prof. Thomas Zeltner: Darum geht es nicht in erster Linie. Ich wurde als eine von rund 20 Personen eingeladen, an einem Programm mitzuarbeiten, das ich für wichtig und sinnvoll halte. Hintergrund ist ein Phänomen, das für unsere Zeit kennzeichnend ist: Wir beobachten eine rasch zunehmende Chancenungleich- heit zwischen Reich und Arm - sowohl auf der Ebene der Länder als auch innerhalb der Nationen. Diese Tendenz betrifft auch den Zugang zu Gesundheitsdienstleistungen. Solche Fragen der sozialen Gerechtigkeit haben mich seit jeher stark bewegt. Ich hoffe, mit meinem Engagement ein klein wenig dazu beitragen zu können, diesem Auseinanderdriften entgegenzuwirken.

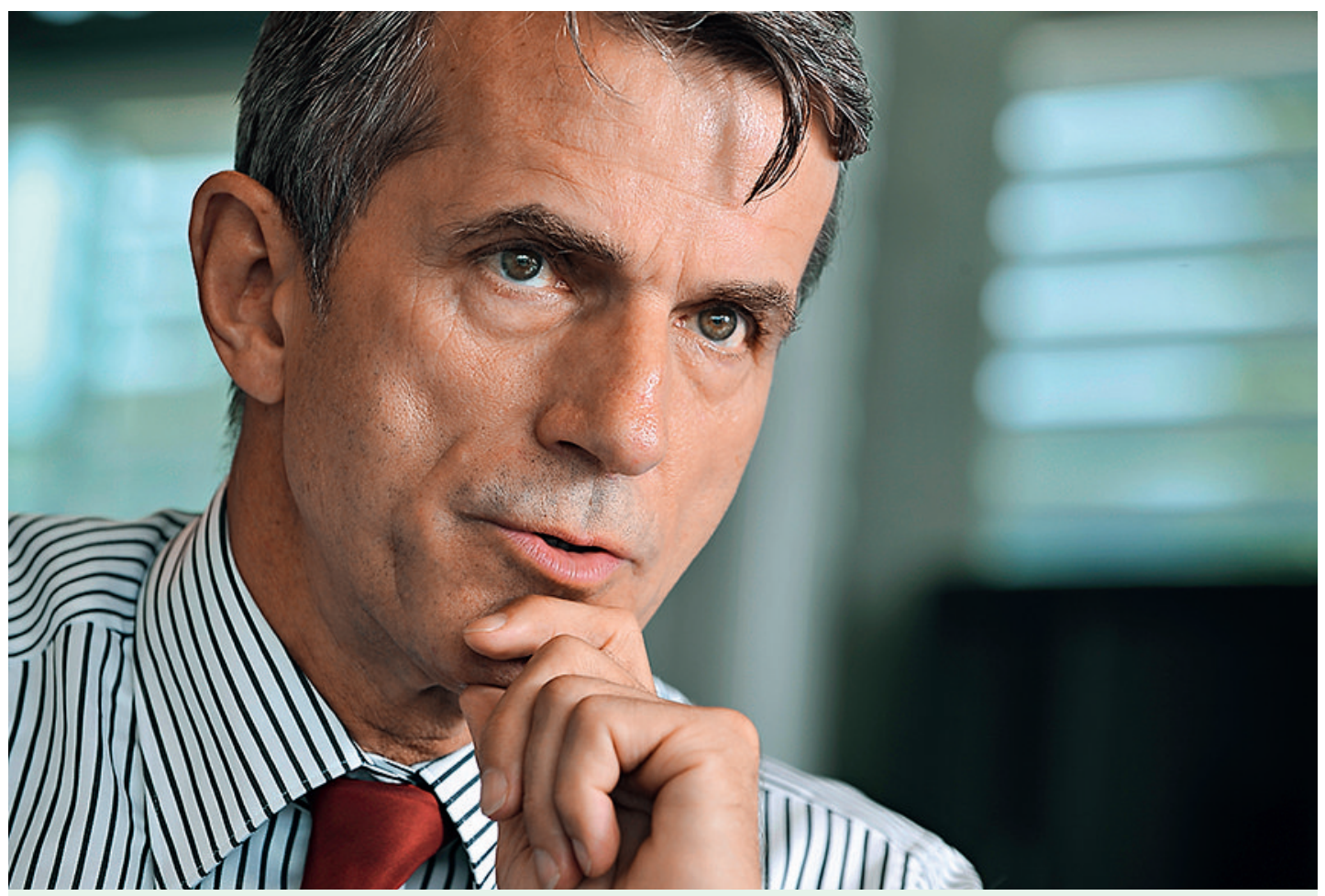

Engagiert sich für soziale Gerechtigkeit im globalen Gesundheitsmarkt: Thomas Zeltner.

Fotos: BAG/Peter Mosimann 
Hat man sich dieses Programm demnach als eine Art «Think Tank» vorzustellen?

Es ist mehr als ein Think Tank. Von den involvierten Personen wird erwartet, dass sie ihre Erfahrungen und ihr Netzwerk auch dazu nutzen, konkrete Programme auf die Beine zu stellen. Einer meiner grössten Pluspunkte - wenn ich das so sagen darf - ist wahrscheinlich, dass ich im Gesundheitsbereich fast «alle» kenne.

\section{«Man kann einen wildgewordenen Markt nicht sich selbst überlassen - das gilt für die Schweiz, in weitaus stärkerem Masse aber international»}

Können Sie das inhaltlich etwas konkretisieren? Es geht teilweise um elementare Dinge: Was wird in welchen internationalen Gremien entschieden, wie verhält man sich in diesen Gremien? Ein sehr wichtiger Bereich ist auch die sich ändernde Zusammenarbeit zwischen Staat und Wirtschaft sowie Fragen der «PPPs», der Public-Private-Partnerships. Konkret könnte ein Programm beispielsweise zum Ziel haben, einem armen Land aufzuzeigen, wie es zu Impfstoffen kommen kann, indem Know-how über Produktion und Distributionskanäle und Finanzierungsmöglichkeiten vermittelt wird.

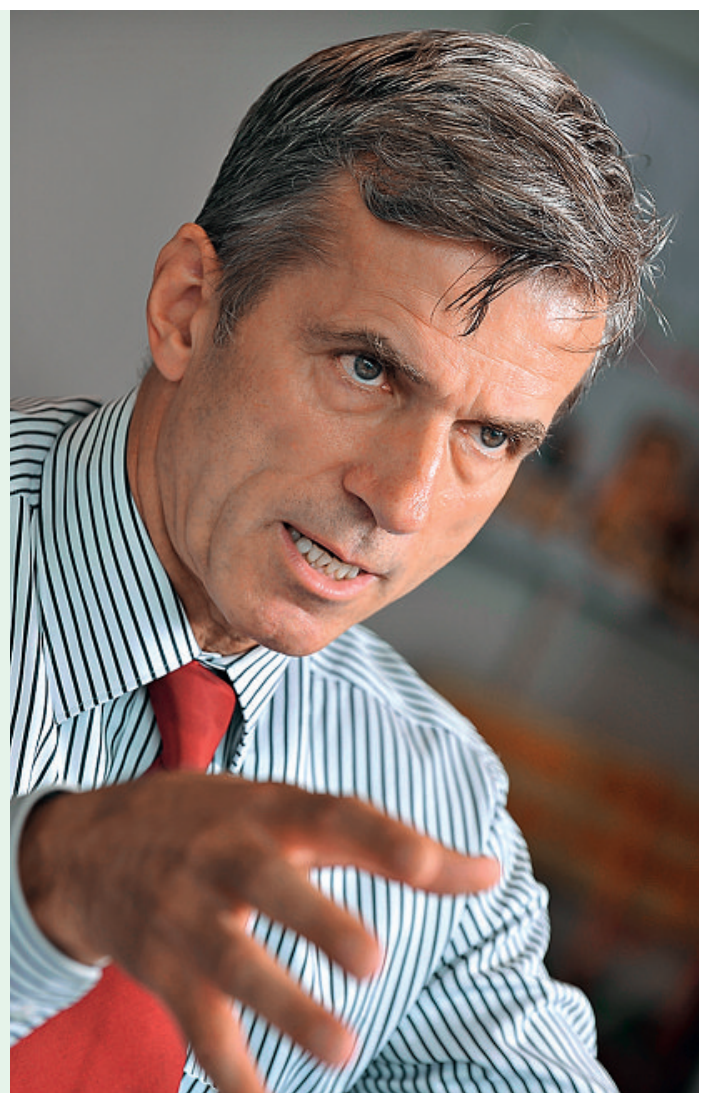

Für Thomas Zeltner haben die Hausärztinnen und Hausärzte weiterhin eine zentrale Stellung in unserem Gesundheitswesen.
Wenn Sie von der Wichtigkeit der «internationalen Gremien» sprechen: Wie hat sich die Globalisierung generell auf das Gesundheitswesen und auf Ihre Arbeit als BAGDirektor ausgewirkt?

Heute haben wir eine total vernetzte Welt, die zugleich in Bezug auf die Gesundheit in den letzten 20 Jahren ein viel unsicherer Platz geworden ist. Sicherheitsfragen im Zusammenhang mit Bioterrorismus, Pandemien, explodierenden Antibiotikaresistenzen, um einige Stichworte zu nennen, haben damit stark an Bedeutung zugenommen. Die Globalisierung führte dazu, dass riesige neue Sicherheitsdispositive aufgezogen werden mussten. Die Überwachung der Gesundheit bis hin zur letzten Spritze, die von der WHO koordiniert in einer Arztpraxis landet - das alles kannte man vor 20 Jahren nicht. Was meine Arbeit betrifft: Früher war die internationale Aktivität des BAG auf eine Person beschränkt. Heute ist dafür eine ganze Abteilung mit einem Dutzend Leuten unter der Leitung eines Vizedirektors zuständig. Die gesamte Arbeit des BAG ist nicht mehr denkbar ohne einen ganz starken Fokus darauf, was in der Welt läuft.

Die Globalisierung hat aber nicht nur die Bedrohungslage verändert.

Sie eröffnet den Anbietern von Dienstleistungen im Gesundheitsbereich, seien dies die Pharmaindustrie, die Pflegenden oder die Ärzteschaft, auch grosse neue Märkte - und damit natürlich immer auch für die Patienten neue Möglichkeiten. Der ganze Gesundheitsmarkt hat sich enorm schnell verändert - die Pharmaindustrie zum Beispiel kann heutzutage gar nicht mehr anders tätig sein als global. Oder nehmen Sie die Karawanen von Angehörigen der Gesundheitsberufe, die heutzutage um den Globus ziehen - solche Dinge sind neu und verlangen nach neuen Regeln.

Wie kann ein weltweiter «Gesundheitsmarkt» geregelt werden?

Viele Dinge sind bereits geregelt, und es kommen laufend neue dazu. Ich glaube daran, dass sich Markt und Wettbewerb sehr positiv auf die Qualität auswirken können, dass sie aber in Bezug auf Chancengleichheit und Zugang auch verheerend sein können. Wenn die Amerikaner und die Europäer eine grosse Zahl an Ärzten aus Afrika oder Indien abziehen, löst das zwar Probleme in der reichen Welt, ist aber nicht vereinbar mit unseren Vorstellungen zu Fairness und Chancengleichheit. Das sind Prinzipien, zu deren Schutz die Staatengemeinschaft als Regulator eingreifen muss. Man kann einen wildgewordenen Markt nicht sich selbst überlassen - das gilt für die Schweiz, in weitaus stärkerem Masse aber international.

Wechseln wir von der internationalen auf die nationale Ebene. Aus Sicht der Ärzteschaft, insbesondere der Grundversorger, war das Vertrauensverhältnis zum BAG in den letzten Jahren stark gestört, die Kommunikation schwierig. 
Das ist leider so. Ich denke aber auch, dass die Schwierigkeiten der Grundversorger zu einem guten Teil dadurch bedingt sind, dass sie zu demjenigen Teil des Gesundheitssystems gehören, der vor einem notwendigen Veränderungsprozess steht. Die grosse Baustelle, in der gegenwärtig Reformen anstehen, ist die ambulante Medizin. Stichworte dazu sind z.B. die integrierte Versorgung, Managed Care usw. Wenn wir diesen Schritt gemacht haben - und das ist harte Arbeit sind wir wieder ganz gut aufgestellt
Wodurch ist diese Vertraulichkeit-oder aus Sicht der Ärzte: Intransparenz - gerechtfertigt?

Wenn die Prozesse völlig transparent sind, machen die verschiedenen Lobbygruppen - und da gehören die Ärzte halt auch dazu - via Öffentlichkeit derart viel Druck auf die Kommissionsmitglieder, dass eine sachliche Debatte in der Kommission fast nicht mehr möglich ist. Das hat man zum Beispiel beim Programm Evaluation Komplementärmedizin (PEK) gesehen, als der Druck auf die Mitglieder der Kom-

\section{«Ich verstehe den Reflex, zu sagen: Jetzt haben sie uns zugehört und machen trotzdem etwas ganz anderes`. Aber genau so sieht es das System vor»}

Die Vorwürfe an das BAG waren aber teilweise sehr konkret. Für die Ärzte war es zum Beispiels äusserst stossend, dass unklar blieb, wie die neuen Labortarife zustande kamen, während sie selbst ja ein transparentes, nachvollziehbares Point-of-Care-Modell vorlegten.

Bei der Analyseliste hatten die Ärzte eine Erwartung, die schlichtweg nicht erfüllbar war. Es gibt wenige Domänen in der Medizin - die Analyseliste ist eine davon - in denen der Staat die Preise einseitig festsetzt. Das ist ein völlig anderer Mechanismus als beim TARMED, der Ergebnis von Verhandlungen war.

Aber hätte man das nicht klarer kommunizieren können, anstatt die Ärzte anzuhören und dann doch «eigenmächtig» zu entscheiden?

Die Festlegung der Tarife durch den Staat ist in unserem System die Ausnahme und deshalb für die mission seitens der Medien und der Komplementärmediziner enorm war. In einer solchen Atmosphäre noch einen ruhigen, sachbasierten, unabhängigen Entscheid zu fällen, ist ohne eine Vertraulichkeit praktisch nicht möglich.

Wie haben Sie in Ihrer Amtszeit die Ärzteschaft als Partner, aber auch als Interessenvertreter wahrgenommen?

Ich habe die Ärzteschaft insgesamt als heterogen wahrgenommen. In der organisierten Ärzteschaft finden sich sehr unterschiedliche Gruppierungen. Der für mich «konfliktfreiste» Partner war in all den Jahren die Schweizerische Akademie der Medizinischen Wissenschaften SAMW, die stark mit Forschung und Wissenschaft verbunden ist. Am spannungsreichsten war das Verhältnis in letzter Zeit tatsächlich mit den ambulanten Grundversorgern.

\section{«Wir möchten eigentlich, dass die Grundversorger in unserem System zu den Gewinnern gehören, was mit dem TARMED leider nicht eingetreten ist»}

Ärzte wenig einsichtig. Die verschiedenen Akteure werden dabei zwar angehört, - was auch bei der Analyseliste der Fall war - verhandelt wird jedoch nicht. Dass man in Sachen Kommunikation einiges hätte besser machen können, bestreite ich nicht. Wenn wir von Transparenz reden, muss ich aber auch auf deren Grenzen hinweisen. Wir haben ein System von Kommissionen, deren Arbeit vertraulich ist und nicht in die Öffentlichkeit gelangen soll. Ich verstehe den Reflex, zu sagen: «Jetzt haben sie uns zugehört und machen trotzdem etwas ganz anderes.» Aber genau so sieht es das System vor.
Empfanden Sie dies persönlich als belastend? Ja, das kann man sagen. Ich hatte zum Beispiel nie ein Problem damit, mit der Tabakindustrie auf Konfrontationskurs zu gehen. Es ist ja klar, dass meine Interessen und deren Interessen, mein Job und ihr Job, in gewissem Sinn inkompatibel sind. Bei den Grundversorgern war die Konstellation für mich sehr viel schwieriger, weil es sich um eine Berufsgruppe handelt, die für die Patienten das Beste will - genau wie wir auch. Die Differenzen liegen also nicht beim Endziel, sondern man ist sich bezüglich des Wegs nicht einig, auf dem das angestrebte Ziel erreicht wird. Der 
Hausarzt, die Hausärztin sind und bleiben aus meiner Sicht aber zentrale Elemente in unserem Gesundheitssystem.

\section{«Die Schweizer Bevölkerung würde massive Einbrüche bezüglich der Versorgungsqualität nie akzeptieren»}

In diesem Punkt scheint politisch ein breiter Konsens zu bestehen. Dennoch ist das Einkommen der freien Ärzteschaft, gemessen am Landesindex der Konsumentenpreise, in den letzten Jahren stets gesunken, während dasjenige der Arbeiter und Angestellten gestiegen ist.

Das ist meiner Ansicht nach eine der bedauerlichen Fehlentwicklungen in unserem Gesundheitswesen. Wir möchten eigentlich, dass die Grundversorger in unserem System zu den Gewinnern gehören, was mit dem TARMED leider nicht eingetreten ist. Um diese Fehlentwicklung zu korrigieren, müsste man aber verschiedene Probleme auf den Tisch legen - auch innerhalb der Ärzteschaft. Es gibt beispielsweise die Problematik, dass die Grundversorger den Spezialisten nicht ins Gärtchen treten wollen. Aus Sicht der Ärzteschaft müsste meiner Meinung nach ernsthaft erwogen werden, den TARMED zu kündigen oder zumindest einschneidend zu verändern.

Welche Alternativen gäbe es denn?

Ich glaube, relativ viele junge Ärzte suchen heute nach neuen Modellen, auch Anstellungsmodellen in der Ambulanz, die vielleicht zur Lösung der durch die Konstruktion TARMED bedingten Schwierigkeiten beitragen können. Integrierte Versorgung und Managed Care könnten durchaus erfolgversprechende Wege sein.
Wäre überhaupt klar, wie man das angehen müsste? Ich glaube schon. Ein Element ist das FallpauschalenSystem DRG, von dem wir uns erhoffen, dass es durch die Vergleichbarkeit der Preise und dadurch mehr Transparenz über eine gewisse Spezialisierung eine qualitative Verbesserung bringen wird. In der ambulanten Medizin ist für mich der Weg integrierte Versorgung, Behandlungspfade, Gatekeeping, Hausarztmodelle.

Sind Sie generell bezüglich der Einführung der DRG optimistisch?

Grundsätzlich bin ich zuversichtlich. Ein rechter Teil der Spitäler arbeitet ja heute schon mit Fallpauschalen- vieles deutet darauf hin, dass dieses System so schlecht nicht funktionieren wird. Natürlich werden sich mit der Einführung wie bei den Ländern, die dieses System schon haben, gewisse Fragen ergeben, die dann wiederum Reformen auslösen werden. Die Schweizer Bevölkerung würde massive Einbrüche bezüglich der Versorgungsqualität aber nie akzeptieren.

Es gibt aber auch renommierte Professoren und Klinikchefs, die befürchten, dass als Folge der DRG Innovationen gebremst und die Patienten nicht mehr die optimale Behandlung erhalten werden.

Ich finde es gut, wenn auf potentielle Probleme hingewiesen wird. Ich habe mich beispielsweise immer gegen die Rationierung von Einzelleistungen ausgesprochen, selbst bei sehr teuren Medikamenten. Wichtig ist aber auch, dass man bei jeder Intervention genau hinschaut: Muss es wirklich immer das absolut Letzte, Teuerste sein? Nach übereinstimmender Meinung der Experten könnten durch Managements- und Prozessverbesserungen 10-15\% der Kosten im Gesundheitswesen eingespart werden. Aber die systemischen Probleme sind immer schwieriger zu beeinflussen als einzelne Leistungen.

\section{«Wenn Programme gegen Tabak- und Alkoholmissbrauch koordiniert ablaufen, dann erzielen sie Wirkung. Davor fürchten sich die betreffenden Wirtschaftskreise»}

Wo sehen Sie weitere Fehlentwicklungen?

Wir haben in diesem Land - im internationalen Vergleich - zu viele kleine Einheiten, zu viele kleine Spitäler, zu viele kleine Arztpraxen. In Bezug auf den Patientenkomfort ist das grossartig, gerade auch für eine älter werdende Bevölkerung. Dagegen gibt es viele Indikatoren, die zeigen, dass diese Situation in Bezug auf die Outcome-Qualität nicht optimal ist. Aufgrund dieser Erkenntnis müsste der Komfort-Level eigentlich etwas zugunsten der Outcome-Qualität verschoben werden. Aber das ist ein sehr, sehr schwieriger Prozess.
Wenn Sie unser Gesundheitswesen insgesamt betrachten: Wo orten Sie das grösste Verbesserungspotential?

Eigentlich sind sich alle Wissenschaftler und auch Regierungen einig, dass der grösste Gesundheitsgewinn oder «Health Gain» durch eine Stärkung von Gesundheitsförderung und Prävention erzielt werden kann.

Mit den HIV-/Aids-Kampagnen und verschiedenen nationalen Aktionsprogrammen haben Sie in der Prävention in Ihrer Amtszeit ein paar entscheidende Pflöcke eingeschla- 
